\title{
Interleukin-18 Synergism with Interleukin-2 in Cytotoxicity and NKG2D Expression of Human Natural Killer Cells
}

\author{
Yuan-Ying Qi, Chao Lu, Ying Ju, Zi-E Wang, Yuan-Tang Li, Ya-Juan Shen*, \\ Zhi-Ming Lu*
}

\begin{abstract}
Natural killer (NK) cells play an important role in anti-tumor immunity. Interleukin (IL)-18 is an immunoregulatory cytokine that induces potent NK cell-dependent anti-tumor responses when administrated with other cytokines. In this study, we explored the effects of combining IL-18 and IL-2 on NK cytotoxicity as well as expression levels of the NK cell receptor NKG2D in vitro. Freshly isolated PBMCs were incubated for $48 \mathrm{~h}$ with IL-18 and IL-2, then CD107a expression on CD3-CD56 ${ }^{+} \mathrm{NK}$ cells was determined by three-colour flow cytometry to evaluate the cytotoxicity of NK cells against human erythroleukemia K562 cells and human colon carcinoma HT29 cells. Flow cytometric analysis was also employed to determine NKG2D expression on NK cells. The combined use of IL-18 and IL-2 significantly increased CD107a expression on NK cells compared with using IL-18 or IL-2 alone, suggesting that the combination of these two cytokines exerted synergistic enhancement of NK cytotoxicity. IL-18 also enhanced NKG2D expression on NK cells when administered with IL-2. In addition, blockade of NKG2D signaling with NKG2D-blocking antibody attenuated the up-regulatory effect of combining IL-18 and IL-2 on NK cytolysis. Our data revealed that IL-18 synergized with IL-2 to dramatically enhance the cytolytic activity of human NK cells in a NKG2D-dependent manner. The results appear encouraging for the use of combined IL-18 and IL-2 in tumor immunotherapy.
\end{abstract}

Keywords: Interleukin-18 - interleukin-2 - natural killer cells - NKG2D - cytotoxicity

Asian Pac J Cancer Prev, 15 (18), 7857-7861

\section{Introduction}

Natural killer (NK) cells are a major component of effector lymphocytes in the early host defenses against tumors and infections as they can directly kill target cells without prior stimulation (Vivier et al., 2008). Activated NK cells also produce inflammatory cytokines and crosstalk with dendritic cells to elicit specific anti-tumor responses (Hu et al., 2013; Wong et al., 2013). NK cells express a variety of surface activating and inhibitory receptors, the engagement of which mediates NK cell activity and determines whether target cells are killed by NK cells or not (Konjevic et al., 2012). Natural killer group 2 , member D (NKG2D) is an activating immunoreceptor expressed by NK and T cells. Several ligands of NKG2D receptor have been identified to date, including human UL16-binding proteins (ULBPs) and MHC I-related A/B (MICA/B) molecules that are frequently up-regulated on abnormal cells but poorly expressed by healthy adult tissue (Huergo-Zapico et al., 2014). By recognizing and binding to corresponding ligands expressed on tumor cells, NKG2D regulates the activation of effector lymphocytes and plays a vital role in cell-mediated tumor surveilance and elimination (Zafirova et al., 2011; Shen et al., 2012; Yu et al., 2014).

NK cell function is also regulated by cytokines (Marcais et al., 2013). Interleukin (IL)-2 enhances NK cell proliferation, cytolytic activity and production of several cytokines such as IFN- $\gamma$, TNF- $\alpha$ and GM-CSF (Gaffen and Liu, 2004). However, a series of severe adverse effects related with high-dose or long-term IL-2 treatment have greatly limited its clinical application in tumor immunotherapy (Atzpodien and Reitz, 2005; Clark et al., 2013). IL-18, originally described as an IFN- $\gamma$-inducing factor, is an immunostimulatory cytokine primarily secreted from activated macrophages and dendritic cells. IL-18 not only exerts a potential effect on the promotion of NK cell function (Srivastava et al., 2013), but also acts as a necessary promoter for NK cells to display full effector activity (Chaix et al., 2008). There is considerable evidence that $\mathrm{IL}-18$ inhibits the growth and metastasis of tumor cells in several mice models of cancer (Tse et al., 2011; Kuppala et al., 2012). Meanwhile, clinical studies have demonstrated that systemic administration 
of IL-18 to patients with advanced cancer can generate a relatively limited anti-tumor efficacy but with no apparent toxicities (Robertson et al., 2008; Tarhini et al., 2009). Because of their divergent but complementary properties, the combination of IL-18 and IL-2 is considered as a viable strategy to induce NK cell-mediated anti-tumor responses (Son et al., 2001). However, the exact regulatory mechanism of combining IL-18 and IL-2 on NK cell function is still not well understood.

In the present study, we investigated the effects of combining IL-18 and IL-2 on cytolytic activity and NKG2D expression of human NK cells in vitro. We assayed NK cytotoxicity by multi-parameter flow cytometry using a marker, lysosomal-associated membrane protein-1 (LAMP-1 or CD107a), which can be expressed on the cell surface following degranulation. We also examined the expression of NKG2D on NK cells by using flow cytometry. We found that IL-18 synergized with IL-2 to promote the cytotoxicity and NKG2D expression of NK cells. The synergistic enhancement of NK cytolysis by both cytokines was significantly attenuated by NKG2D blockade. Therefore, we concluded that IL-18 acted synergistically with IL-2 to enhance NK cell activity at least partly via NKG2D pathway.

\section{Materials and Methods}

\section{Cytokines and antibodies}

Recombinant human IL-18 (rhIL-18) was purchased from R\&D systems (Minneapolis, MN, USA) and rhIL-2 was from Shanghai Sangon Biotech Co., Ltd (Shanghai, China). Both cytokines were reconstituted in sterile distilled water and were endotoxin free. PE-conjugated mouse anti-human CD3, APC-conjugated mouse antihuman CD56, and FITC-conjugated mouse anti-human CD107a monoclonal antibodies were products of BD Pharmingen (San Diego, CA, USA). Alexa Fluor ${ }^{\circledR}$ 488-conjugated mouse anti-human NKG2D monoclonal antibody ( $\mathrm{mAb}$ ) and neutralizing antibody against NKG2D (anti-human NKG2D-blocking mAb, mouse IgG1) were purchased from R\&D systems (Minneapolis, MN, USA).

\section{PBMCs isolation and cytokine stimulation}

Peripheral blood samples were obtained from healthy donors. Peripheral blood mononuclear cells (PBMCs) were isolated from heparinized venous blood in sterile by Ficoll-Histopaque density gradient centrifugation (Tianjin Haoyang, China). These cells were washed two times with RMPI 1640 medium (Hyclone, Logan, UT, USA) and were resuspended at a density of $1 \times 10^{6}$ cells $/ \mathrm{ml}$ in RMPI 1640 medium containing 10\% fetal bovine serum (FBS), $100 \mathrm{U} / \mathrm{ml}$ streptomycin and $100 \mathrm{U} / \mathrm{ml}$ penicillin. The cells were placed in a 96-well sterile cell culture plate (Costar, Corning, NY, USA) and stimulated with various concentration combinations of rhIL-18 $(0,100$, $200 \mathrm{ng} / \mathrm{ml})$ plus rhIL-2 $(0,100,200 \mathrm{U} / \mathrm{ml})$ for $48 \mathrm{~h}$. Three replicate wells were used. All cultures were maintained in a humidified incubator with $5 \% \mathrm{CO}_{2}$ at $37^{\circ} \mathrm{C}$.

\section{Assay of CD107a degranulation}

Freshly isolated or cytokine-stimulated PBMCs were cocultured with human erythroleukemia K562 cells or human colon carcinoma HT29 cells in a 96-well cell culture plate with an effector/target (E:T) ratio of 10:1. The final volume was $100 \mu \mathrm{l}$ in each well. The cells were then stained with FITC-conjugated anti-CD107a mAb and incubated for $5 \mathrm{~h}$ in a humidified incubator with $5 \%$ $\mathrm{CO}_{2}$ at $37^{\circ} \mathrm{C}$. Monensin (eBioscience, San Diego, CA, USA) was added to each well at a final concentration of $2 \mu \mathrm{mol} / \mathrm{l}$ during the last $4 \mathrm{~h}$ of the culture to prevent the degradation of CD107a from NK cell surface and ensure the detectability of this marker after stimulation. After $5 \mathrm{~h}$ incubation, the cells were stained with PE-conjugated antiCD3 mAb and APC-conjugated anti-CD56 mAb. CD107a expression on $\mathrm{CD} 3 \mathrm{CD}^{-} 6^{+} \mathrm{NK}$ cells was determined on a FACS Calibur flow cytometer (BD Bioscience, San Jose, CA, USA).

To verify the relationship between NK cytolytic enhancement and NKG2D expression on NK cells, untreated or stimulated PBMCs were pre-incubated with NKG2D-blocking mAb or isotype control IgG for $1 \mathrm{~h}$ before the coculture with target cells. Then, CD107a degranulation assays were performed.

\section{Flow cytometric analysis of $N K G 2 D$ expression}

After different stimulations, cells were collected, washed and resuspended in $100 \mu \mathrm{l}$ staining buffer (PBS with $0.5 \%$ BSA and $0.1 \%$ sodium azide) at $1 \times 10^{6}$ cells/ $\mathrm{ml}$. Subsequently, these cells were incubated with PEconjugated anti-CD3 mAb, APC-conjugated anti-CD56 $\mathrm{mAb}$, and Alexa Fluor ${ }^{\circledR}$ 488-conjugated anti-NKG2D mAb at $4^{\circ} \mathrm{C}$ in the dark for $30 \mathrm{~min}$, washed twice, resuspended in $300 \mu \mathrm{l}$ staining buffer directly for flow cytometric analysis. NKG2D expression on $\mathrm{CD}^{-} \mathrm{CD}^{-} 6^{+} \mathrm{NK}$ cells was examined on a FACS Calibur flow cytometer (BD Bioscience, San Jose, CA, USA).

\section{Statistical analysis}

Student's t test was used to analyze whether there were significant differences in cytotoxicity and NKG2D expression of $\mathrm{NK}$ cells between the combined use of cytokines (IL-18 and IL-2) and either cytokine alone (IL18 or IL-2). A value of $p<0.05$ was regarded as statistically significant. Statistical analyses were performed by using GraphPad Prism 5 (GraphPad Software, USA).

\section{Results}

IL-18 synergized with IL-2 to improve CD107a degranulation of human $\mathrm{NK}$ cells in vitro

When cytotoxicity assay was performed against K562 cells, both IL-18 and IL-2 clearly promoted the expression of CD107a on CD3-CD56 ${ }^{+} \mathrm{NK}$ cells. Compared with using IL-18 or IL-2 alone, the combination of IL-18 (200 ng/ml) and IL-2 $(100 \mathrm{U} / \mathrm{ml})$ significantly increased the percentage of CD107a positive NK cells (24.89\% versus $4.18 \%$ and $5.63 \%$, respectively; $p<0.01$; Figure $1 \mathrm{~A}$ ). Similar results were also observed when using HT29 cells as targets of cytotoxicity test (Figure 1A). Besides, CD107a expression on the surface of NK cells was up-regulated by IL-18 plus IL-2 in a dose-dependent manner (Figure 1B). Although NK cells might have different sensitivity to K562 and 
HT29 cells, our results revealed that IL-18 dramaticlly enhanced the cytolytic activity of NK cells against these two tumor cells when combined with IL-2.

\section{IL-18 and IL-2 did not affect the proliferation of human NK cells}

Isolated PBMCs were cultured for $48 \mathrm{~h}$ in the presence of IL-18 (200 ng/ml) plus IL-2 $(100 \mathrm{U} / \mathrm{ml})$, or either cytokine alone. We observed the effect of combining IL-18 and IL-2 on the expansion of NK cells in vitro by flow cytometric analysis of $\mathrm{CD} 3 \mathrm{CD}^{-} 6^{+}$cell population. As shown in Figure 2, we did not observe significant increases in the percentage of CD3 $\mathrm{CD}^{-} 6^{+} \mathrm{NK}$ cells following different cytokine stimulations. The combined use of IL-18 and IL-2 did not affect the proliferation of human NK cells.
$I L-18$ synergized with IL-2 to increase NKG2D expression
on human NK cells

To explore the regulatory mechanism of combining IL-18 and IL-2 on NK cytolysis, we also observed the expression level of NKG2D receptor, which plays a critical role in NK-mediated cytolysis of target cells. Isolated PBMCs were incubated for $48 \mathrm{~h}$ with IL-18 (200 ng/ $\mathrm{ml})$, IL-2 $(100 \mathrm{U} / \mathrm{ml})$ or a combination of both cytokines. Flow cytometric analysis showed that the combined administration of IL-18 and IL-2 significantly enhanced the expression of NKG2D on CD3-CD56 ${ }^{+} \mathrm{NK}$ cells in comparison with either IL-18 or IL-2 alone $(76.15 \%$ versus $59.88 \%$ and $65.60 \%$, respectively; $p<0.05$; Figure $3 \mathrm{~A}$ and $\mathrm{B})$.

The up-regulatory effect of combining IL-18 and IL-2 on

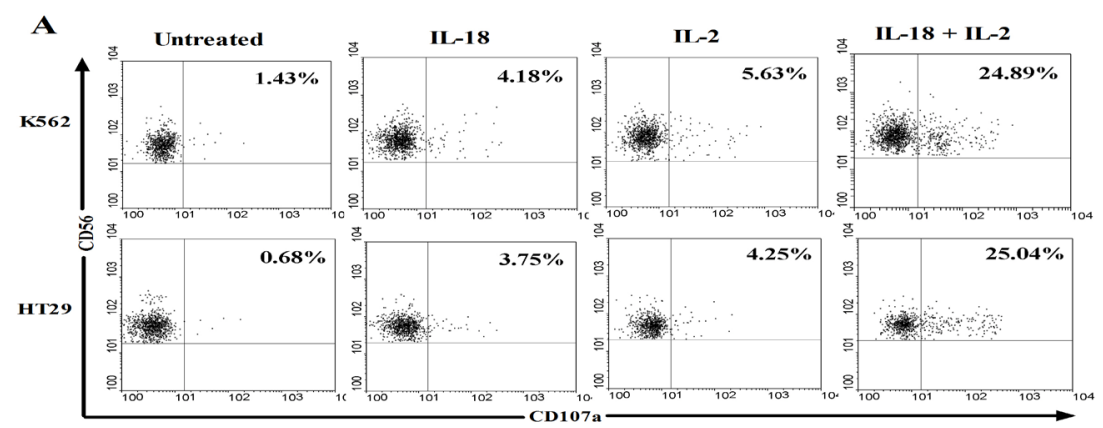

B

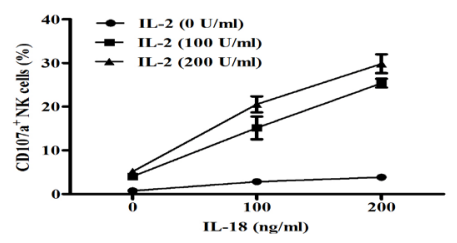

Figure 1. IL-18 Synergized with IL-2 to Increase CD107a Expression on NK Cells. (A) Representative graphs of CD107a expression on gated CD3CD56+ NK cells following no stimulation, stimulation with IL-18 (200 ng/ml), IL-2 (100 U/ $\mathrm{ml}$ ) or a combination of both cytokines. NK cytolysis was assayed against K562 and HT29 cells, respectively. Similar results were observed in three independent experiments. (B) The line charts represent the expression pattern of CD107a on NK cells after the culture with different dose combinations of IL-18 and IL-2. Data are given as the mean \pm SD from three independent experiments
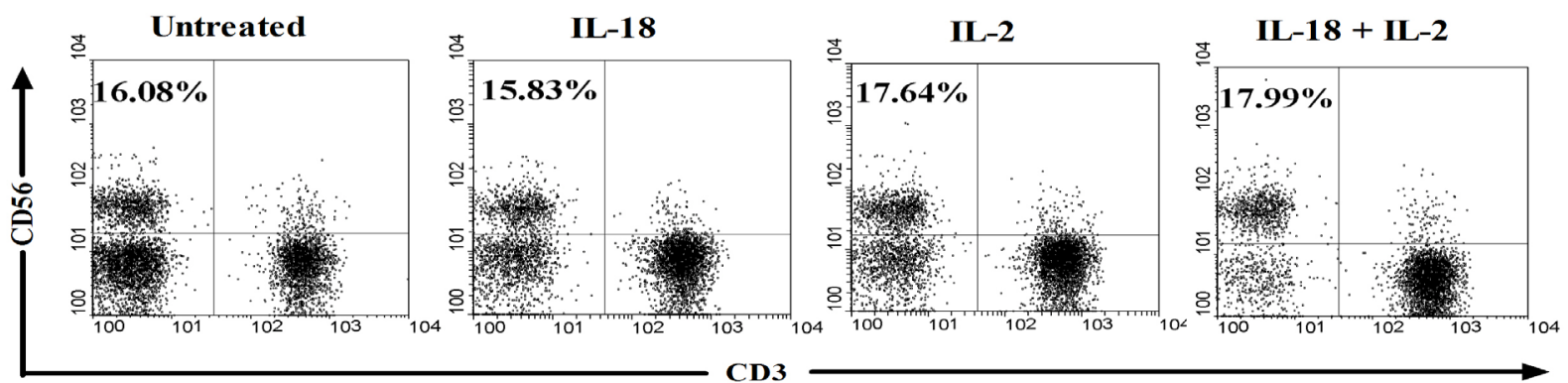

Figure 2. IL-18 and IL-2 Did Not Affect the Proliferation of Human NK Cells. Flow cytometric analysis of the expansion of CD3 CD56 ${ }^{+} \mathrm{NK}$ cells following no stimulation, stimulation with IL-18 $(200 \mathrm{ng} / \mathrm{ml})$, IL-2 $(100 \mathrm{U} / \mathrm{ml})$ or a combination of both cytokines. Representative results of three independent experiments are shown here

$\mathbf{A}$

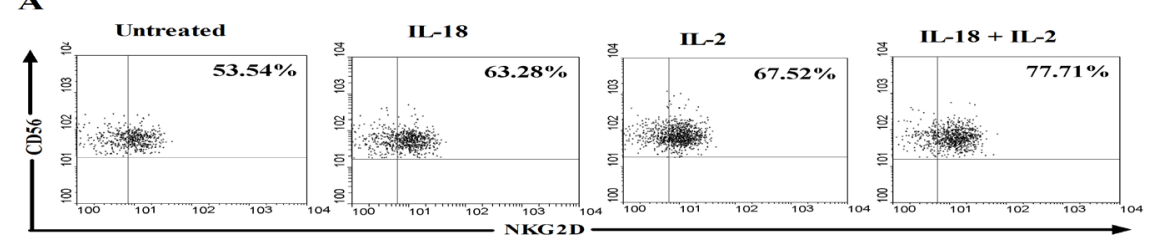

B

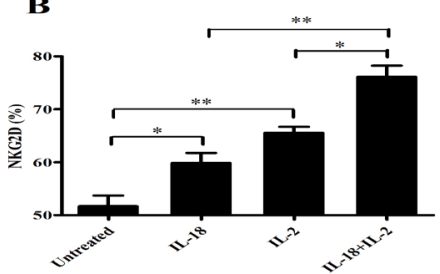

Figure 3. IL-18 Synergized with IL-2 to Enhance NKG2D Expression on NK Cells. NKG2D expression levels on NK cells determined by flow cytometry were compared following no stimulation, stimulation with IL-18 (200 ng/ml), IL-2 (100 U/ $\mathrm{ml}$ ) or a combination of both cytokines. (A) Representative graphs of NKG2D expression on gated CD3 CD56 ${ }^{+} \mathrm{NK}$ cells. (B) The bar graphs represent the expression pattern of NKG2D on NK cells after different stimulations. Data are given as the mean \pm SD of three independent experiments $(* P<0.05$ and $* * P<0.01)$ 
$\mathbf{A}$

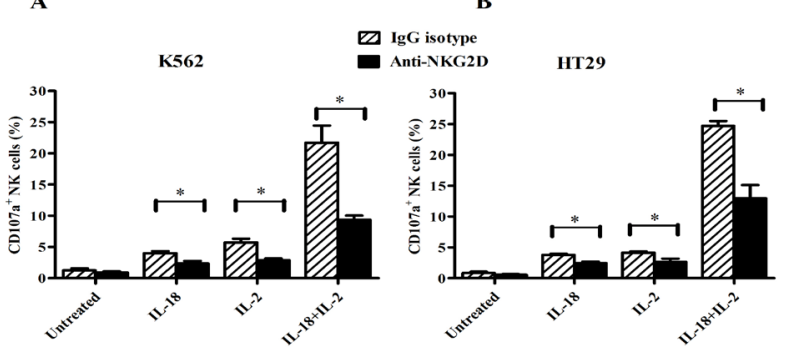

Figure 4. NK Cytolytic Enhancement by IL-18 Plus IL-2 was Dependent on the Up-regulatory Expression of NKG2D. NK cytolysis against K562 (A) and HT29 (B) cells was determined by flow cytometric analysis of the percentage of CD107a positive NK cells after pre-incubation of isolated or stimulated cells with anti-NKG2D mAb. Data represent the mean $\pm \mathrm{SD}$ of three independent experiments. ${ }^{*} \mathrm{P}<0.01$ versus $\mathrm{IgG}$ isotype control group

\section{$N K$ cytotoxicity was $N K G 2 D$-dependent}

We next blocked NKG2D pathway with anti-NKG2D antibody to investigate the role of NKG2D in the regulation of NK cytotoxicity by IL-18 and IL-2. We observed that the blockade of NKG2D receptor significantly inhibited NK cell-mediated lysis of target cells. The up-regulatory effects of combining IL-18 and IL-2 on NK cytolysis against K562 and HT29 cells were dramatically attenuated by NKG2D blockade (Figure 4). These results suggested that IL-18 synergized with IL-2 to improve the cytolytic activity of NK cells at least partly via NKG2D pathway.

\section{Discussion}

NK cells are increasingly considered as potential targets for tumor immunotherapy. Syngeneic IL-12/ IL-15/IL-18-pretreated NK cells can proliferate rapidly in tumor tissue and induce sustained anti-tumor effects after adoptive transfer to tumor-bearing mice ( $\mathrm{Ni}$ et al., 2012). Infusion of continuously growing NK92 cells into patients with lung cancer appears to have some anti-tumor responses without significant side effects (Tonn et al., 2013). Thus, it is necessary to implement a viable strategy to improving NK cell activities so as to strengthen human's anti-tumor responses.

Both IL-18 and IL-2 are potent activators of NK cell effector function. Although IL-2 has ever been widely used for treatment of patients with advanced cancer, its clinical utility has been greatly limited by the emergence of significant toxicities associated with high-dose or longterm therapy (Atzpodien and Reitz, 2005; Clark et al., 2013). IL-18 is a relatively nontoxic cytokine (Robertson et al., 2008) that promotes the proliferation of activated $\mathrm{T}$ cells, activation of NK cells and cytokine production (Srivastava et al., 2013). Du et al. (2012) have constructed a human IL18-IL2 fusion protein and confirmed that this protein can induce significant NK cell-dependent antitumor reponses in different tumor-bearing mice models.

To further illuminate the role of combining IL-18 and IL-2 in the regulation of NK cell activity, we first investigated the effects of these two cytokines on NK cytotoxicity. Instead of traditional isotope-release assay, NK cytolysis was determined by flow cytometric analysis of the exposure of CD107a onto the cell surface as a result of degranulation (Aktas et al., 2009). We found that unstimulated NK cells had a very low level of cell surface CD107a expression and that CD107a expression was significantly up-regulated by IL-18 or IL-2 stimulation. When both cytokines were administrated at the same time, the percentage of CD107a positive NK cells increased approximately 4- and 6-fold compared with using IL-2 or IL-18 alone, suggesting that the cytolytic activity of NK cells was dramatically enhanced. Similar results were observed whether K562 or HT29 was used as targets of cytotoxicity assay. However, the combination of IL-18 and IL-2 did not affect the proliferation of NK cells. These data are somewhat inconsistent with a previous report from Son et al. (2001).

NKG2D is a key activating receptor expressed on the surface of almost all NK cells and is extremely important in the recognition and elimination of tumor cells (Shen et al., 2012; Zhao et al., 2014). Recent researches have demonstrated that both IL-12 and IL-15 can augment the cytolytic activity of NK cells by up-regulating the surface expression of NKG2D on NK cells (Zhang et al., 2008; Tang et al., 2013). In contrast, some cytokines, produced in response to certain danger signals, exert a markedly inhibitory effect on NKG2D expression of NK cells, thereby reducing NK cytolysis against target cells (Muntasell et al., 2010). Besides, IL-18/IL-2 can recover reduced NK cytotoxicity through preventing TGF- $\beta$ induced down-regulation of NKG2D (Song et al., 2006). On the basic of these studies, we hypothesized a possible relationship between NKG2D expression and NK cytolytic enhancement by IL-18 plus IL-2. We found that IL-18 synergized with IL-2 to increase NKG2D expression on NK cells and that blockade of NKG2D with anti-NKG2D $\mathrm{mAb}$ dramatically attenuated the up-regulatory effect of combining IL-18 and IL-2 on NK cytolysis. However, we also observed that NK cytolysis against target cells was not completely eliminated by NKG2D blockade. It might be explained by the existence of other NK cell receptors mediating NK cell activity. These results demonstrated that the synergistic enhancement of NK cytotoxicity by IL-18 plus IL-2 was at least partly dependent on the upregulation of NKG2D expression.

Cytokine therapy has been widely considered as a promising strategy for cancer immunotherapy. For instance, IL-21 and IL-7 could exert potent anti-tumor capacities through promoting CIK or CD8+T cellmediated killing of tumor cells (Rajbhandary et al., 2013; Yuan et al., 2014). In the present study, we point out that there is a potential model for tumor immunotherapy based on the improvement of NK cell activity by IL-18 plus IL-2. Studies indicate that the combination of IL-18 and IL-2, or a fusion protein IL-18/IL-2 can induce effective antitumor responses in several tumor-bearing mice models (Son et al., 2003; Du et al., 2012). However, daily injection of IL-18 and IL-2 also leads to severe pulmonary injury in normal mice (Segawa et al., 2011), suggesting that improper combined use of IL-18 and IL-2 may produce undesired physiological consequences. Therefore, further study is needed to evaluate the efficiency and toxicity of these cytokines in clinical application. 
In summary, IL-18 acted synergistically with IL-2 to improve the cytolytic activity of human NK cells in vitro. Substantial up-regulation of NKG2D expression on the surface of NK cells may partly explain the synergistic enhancement of NK cytolysis by both cytokines. Our findings provide an important experimental basis for cancer immunotherapy. Appropriate administration of IL-18 and IL-2 might be a viable approach to induce therapeutic NK cell-mediated anti-tumor responses.

\section{Acknowledgements}

This study was supported by grants from Shandong Natural Science Foundation (No. ZR2013HM057) and Shandong Medicine and Health Technology Development Program (No. 2009QZ022). We declare that we have not any conflicts of interest. We also thank the participants in this work for their involvement and cooperation.

\section{References}

Aktas E, Kucuksezer UC, Bilgic S, et al (2009). Relationship between CD107a expression and cytotoxic activity. Cell Immunol, 254, 149-54.

Atzpodien J, Reitz M (2005). Metastatic renal carcinoma longterm survivors treated with s.c. interferon-alpha and s.c. interleukin-2. Cancer Biother Radiopharm, 20, 410-6.

Chaix J, Tessmer MS, Hoebe K, et al (2008). Cutting edge: priming of NK cells by IL-18. J Immunol, 181, 1627-31.

Clark JM, Kelley B, Titze J, et al (2013). Clinical and safety profile of high-dose interleukin-2 treatment in elderly patients with metastatic melanoma and renal cell carcinoma. Oncology, 84, 123-6.

Du G, Ye L, Zhang G, et al (2012). Human IL18-IL2 fusion protein as a potential antitumor reagent by enhancing NK cell cytotoxicity and IFN-gamma production. J Cancer Res Clin Oncol, 138, 1727-36.

Gaffen SL, Liu KD (2004). Overview of interleukin-2 function, production and clinical applications. Cytokine, 28, 109-23.

Hu YX, Li M, Jia XH, et al (2013). HPV16 CTL epitope peptideactivated dendritic cell and natural killer co-culture for therapy of cervical cancer in an animal model. Asian Pac J Cancer Prev, 14, 7335-8.

Huergo-Zapico L, Acebes-Huerta A, Lopez-Soto A, et al (2014). Molecular bases for the regulation of NKG2D ligands in cancer. Front Immunol, 5, 106.

Konjevic G, Jurisic V, Jovic V, et al (2012). Investigation of NK cell function and their modulation in different malignancies. Immunol Res, 52, 139-56.

Kuppala MB,Syed SB,Bandaru S, et al (2012).Immunotherapeutic approach for better management of cancer--role of IL-18. Asian Pac J Cancer Prev, 13, 5353-61.

Marcais A, Viel S, Grau M, et al (2013). Regulation of mouse NK cell development and function by cytokines. Front Immunol, 4, 450.

Muntasell A, Magri G, Pende D, et al (2010). Inhibition of NKG2D expression in NK cells by cytokines secreted in response to human cytomegalovirus infection. Blood, 115, 5170-9.

Ni J, Miller M, Stojanovic A, et al (2012). Sustained effector function of IL-12/15/18-preactivated NK cells against established tumors. J Exp Med, 209, 2351-65.

Rajbhandary S, Zhao MF, Zhao N, et al (2013). Multiple cytotoxic factors involved in IL-21 enhanced antitumor function of CIK cells signaled through STAT-3 and STAT5b pathways. Asian Pac J Cancer Prev, 14, 5825-31.

Robertson MJ, Kirkwood JM, Logan TF, et al (2008). A doseescalation study of recombinant human interleukin-18 using two different schedules of administration in patients with cancer. Clin Cancer Res, 14, 3462-9.

Segawa S, Goto D, Yoshiga Y, et al (2011). Involvement of NK 1.1-positive gammadeltaT cells in interleukin-18 plus interleukin-2-induced interstitial lung disease. Am J Respir Cell Mol Biol, 45, 659-66.

Shen Y, Lu C, Tian W, et al (2012). Possible association of decreased NKG2D expression levels and suppression of the activity of natural killer cells in patients with colorectal cancer. Int J Oncol, 40, 1285-90.

Son YI, Dallal RM, Lotze MT (2003). Combined treatment with interleukin-18 and low-dose interleukin-2 induced regression of a murine sarcoma and memory response. J Immunother, 26, 234-40.

Son YI, Dallal RM, Mailliard RB, et al (2001). Interleukin-18 (IL-18) synergizes with IL-2 to enhance cytotoxicity, interferon-gamma production, and expansion of natural killer cells. Cancer Res, 61, 884-8.

Song H, Hur DY, Kim KE, et al (2006). IL-2/IL-18 prevent the down-modulation of NKG2D by TGF-beta in NK cells via the c-Jun N-terminal kinase (JNK) pathway. Cell Immunol, 242, 39-45.

Srivastava S, Pelloso D, Feng H, et al (2013). Effects of interleukin-18 on natural killer cells: costimulation of activation through $\mathrm{Fc}$ receptors for immunoglobulin. Cancer Immunol Immunother, 62, 1073-82.

Tang F, Sally B, Ciszewski C, et al (2013). Interleukin 15 primes natural killer cells to kill via NKG2D and cPLA2 and this pathway is active in psoriatic arthritis. PLoS One, 8 , e76292.

Tarhini AA, Millward M, Mainwaring P, et al (2009). A phase 2 , randomized study of SB-485232, rhIL-18, in patients with previously untreated metastatic melanoma. Cancer, 115, 859-68.

Tonn T, Schwabe D, Klingemann HG, et al (2013). Treatment of patients with advanced cancer with the natural killer cell line NK-92. Cytotherapy, 15, 1563-70.

Tse BW, Russell PJ, Lochner M, et al (2011). IL-18 inhibits growth of murine orthotopic prostate carcinomas via both adaptive and innate immune mechanisms. PLoS One, 6, e24241.

Vivier E, Tomasello E, Baratin M, et al (2008). Functions of natural killer cells. Nat Immunol, 9, 503-10.

Wong JL, Berk E, Edwards RP, et al (2013). IL-18-primed helper NK cells collaborate with dendritic cells to promote recruitment of effector CD8+ T cells to the tumor microenvironment. Cancer Res, 73, 4653-62.

Yu DP, Han Y, Zhao QY, et al (2014). CD3+ CD4+ and CD3+ CD8+ lymphocyte subgroups and their surface receptors NKG2D and NKG2A in patients with non-small cell lung cancer. Asian Pac J Cancer Prev, 15, 2685-8.

Yuan CH, Yang XQ, Zhu CL, et al (2014). Interleukin-7 enhances the in vivo anti-tumor activity of tumor-reactive CD8+ T cells with induction of IFN-gamma in a murine breast cancer model. Asian Pac J Cancer Prev, 15, 265-71.

Zafirova B, Wensveen FM, Gulin M, et al (2011). Regulation of immune cell function and differentiation by the NKG2D receptor. Cell Mol Life Sci, 68, 3519-29.

Zhang C, Zhang J, Niu J, et al (2008). Interleukin-12 improves cytotoxicity of natural killer cells via upregulated expression of NKG2D. Hum Immunol, 69, 490-500.

Zhao L, Wang WJ, Zhang JN, et al (2014). 5-Fluorouracil and interleukin-2 immunochemotherapy enhances immunogenicity of non-small cell lung cancer A549 cells through upregulation of NKG2D ligands. Asian Pac J Cancer Prev, 15, 4039-44. 